\title{
DEVELOPMENT OF A LOCATION-BASED SERVICE FOR A BRNO CITY DISTRICT
}

\author{
Jaromír Landaㄹ, Ivo Pisařovic ${ }^{1}$, Jan Kolomazník ${ }^{1}$, \\ Ondřej Švehlaํ, David Procházka
}

\begin{abstract}
${ }^{1}$ Department of Informatics, Faculty of Business and Economics, Mendel University in Brno, Zemědělská 1, 61300 Brno, Czech Republic
\end{abstract}

To cite this article: LANDA JAROMÍR, PISAŘOVIC IVO, KOLOMAZNÍK JAN, ŠVEHLA ONDŘEJ, PROCHÁZKA DAVID. 2018. Development of a Location-Based Service for a Brno City District. Acta Universitatis Agriculturae et Silviculturae Mendelianae Brunensis, 66(5): 1295-1299.

To link to this article: https://doi.org/10.11118/actaun201866051295

\begin{abstract}
There are many different types of places in any city. These places include playgrounds, dog runs, cultural heritage sites and many more. A city usually wants to provide information about location and equipment of these places. The common way is via a city website. However, nowadays a much more common source of information are mobile applications. This article deals with a development of a mobile application for the Brno-North district of Brno city. The application is designed to inform the citizens of the city district about interesting places in their vicinity. The central element of the application is a map which shows where all the interesting places are located. The application is focused primarily on families; however, it can be useful for any citizen of the district.
\end{abstract}

Keywords: Brno, family, Android, iOS, map, mobile application, location based service

\section{INTRODUCTION}

There are many mobile applications designed for citizens. These mobile applications became very popular with a rise of Smart City initiative. It is common that any large city provides its own application for its citizens. Cities like Barcelona (Scott, 2014) and London (Crowley et al., 2018) are very active in becoming Smart Cities. These applications have many different forms from traffic application which shows current traffic in the city ${ }^{1}$ to applications showing interesting places in the city ${ }^{2}$. The city of Brno is not an exception. There are many applications for citizens of Brno, such as
Easy Brno, Naše Brno or Doprava-Brno.cz. Recently, even many city districts decided to have their own mobile applications.

This article describes the development of a mobile application for the Brno-north city district. One of the key district areas of interest is a family. For this reason, the district decided to create a mobile application primarily for families. The application is a part of Family Friendly Audit, which provides support for municipalities in their effort to improve conditions of the family life ${ }^{3}$. The idea behind the mobile application is to show citizens of the city district places important for the family life, such as children playgrounds. The district decided to

1 https://play.google.com/store/apps/details?id=cz.intens.edits.mobileapp.droid

2 https://play.google.com/store/apps/details?id=cz.modrebrno

3 https://www.auditfamilyfriendlycommunity.cz/?change_language=com 
create native apps for two most common platforms, Android and iOS. The name of the app is Průvodce severem (Guide through Brno-North).

\section{Review}

When developing a mobile application, the developers have several options how to develop and release it. The first option is a native application. This is the most common type. Native applications are developed for a single platform. For Android, it usually means development using Android Studio and for iOS development using the Xcode. However, there are many other options, such as creating an application with a built-in web browser. Such application just shows a web page. The advantage of such solution is that it is easily developed. The disadvantage is that it usually does not have the visual specifications for a specific platform and limited functionality. Another option is usage of tools like Xamarin ${ }^{4}$. It allows to develop cross-platform applications that can be deployed on any platform, even on desktop computers (Bilgin, 2016).

The mobile application for citizens can have many different forms. It can be e.g. an augmented reality application (Lee et al., 2012). These applications are becoming very popular. Another type of application is a tourist guide ${ }^{5}$. These guides can play a big role in the development of tourism (Dongwook and Sungbum, 2017).

In general, the applications for citizens can be divided into several categories:

- Traffic applications - these applications provide current information about the traffic in the city. In Brno, an example of such application is the https://www.doprava-brno.cz/.

- News applications - news applications inform citizens what is happening in their city. Popular application in Czechia is e.g. V obraze ${ }^{6}$.

- Places applications - the goal of these applications is to show the citizens interesting places in the city. As mentioned before, there are many mobile applications developed directly for city residents. In the Czech Republic, Česká obec ${ }^{7}$ is a very popular application. The main idea behind the application is to have one app for all the cities. The user can then choose which city he lives in and will receive updates about that particular city or a city district. Another application called inCity ${ }^{8}$ or already mentioned Vobraze work on the same principle.

A specific category of applications and the direct competitors of our application are mobile applications that focus on children playgrounds. The application Ven každý den is designed to show children playgrounds and also the tips for trips. The application focuses on the entire Czech Republic. For this reason, the application shows only around 15 playgrounds in the Brno-north city district. However, the city district has more than 70 playgrounds and sports fields. Another example of an app about playgrounds is a Playground Locator 9 . The app allows to get playgrounds located near the user, but no others. The user is provided with only a couple of playgrounds and no way of getting the rest of them. The application seems to get the data from Google Places API (Google, 2018). The scope of the application is the entire world. My playgrounds ${ }^{10}$ is another example of such kind of application.

Certainly, key source of information is the Google Maps application. However, this is again a source for the entire world. The main advantage of our application is a focus on a single city district which allows us to provide accurate and up to date content for the users.

\section{MATERIALS AND METHODS}

The main motivation of the city district for providing the mobile application was to show its citizens where all the children playgrounds and sports fields are located. However, during the process, the district decided to share much more information important for its residents, specifically for families. The main requirements on the application were:

- The application must show playgrounds, dog runs and organizations located in the district.

- Each place must be shown in the map and also in the list.

- The User must be able to search in the places.

- For any place, the application must show detailed information and show optional images.

- The User must be able to mark a place as a favorite one.

The district decided to implement the application for both Android and iOS operating systems. The development of the application had two challenges: data collection and data storage and updates.

\section{Data creation}

At the beginning of the application development, the district did not have any central data source where all the necessary information could be found. For this reason, the first task was to collect all necessary data. The data about children

\footnotetext{
4 https://www.xamarin.com/

5 https://play.google.com/store/apps/details?id=cat.triangle.bcn\&feature=search_result

https://play.google.com/store/apps/details?id=cz.onlineteam.ocelot\&hl=cs

http://www.ceskaobec.cz/

https://play.google.com/store/apps/details?id=cz.intelis.mobile.smartcity.android

9 https://play.google.com/store/apps/details?id=de.pnpq.playgroundlocator

10 https://play.google.com/store/apps/details?id=beungoud.mypark
} 
playgrounds were collected by students as a project in Geographical information systems course at FBE MENDELU. The students were divided into teams. Each team collected information about several playgrounds. These data were subsequently merged together to create a basis for the application. The main outcome of the measurements were the precise locations of playgrounds. These were not available before. Nonetheless, the most important task was to collect information about organizations. The district employees needed to get the information from all the organizations, which took time.

Very important part of the data aggregation process was to specify the categories for the places. Each place has one primary category, e.g. playground, dog run, and multiple secondary categories. The categories were created to ease the search for specific place and also reflect the structure given by Audit Family Friendly. For instance, the primary category is education and secondary categories are: for pregnant women, for elderly, for young people of age 15 to 26 and many more.

\section{Data storage}

The second challenge was to choose a suitable solution for storing data. Because of the project limitations, the use of server data storage was not possible. The implementation and maintenance of a cloud service for data distribution would lead to substantial costs. For this reason, another way to keep the data updated needed to be found. Moreover, the most important aspect was to provide the municipality an easy way of data maintenance. The decision was made to use KML files created using Google Maps. The key advantage of such solution is that there is no need for any server infrastructure on the side of the municipality; still, the officials can use user-friendly interface of the Google Maps service. The process of data maintenance is:

- For each type of place, a Google Map is created.

- The district fills in the information directly to the Google Map.
- The map is exported to a KML file.

- The file is stored into our mobile application.

Application with a new file is distributed via Google Play Store or App Store.

\section{RESULTS}

This part describes the implementation of our mobile application. The implementation is divided into two parts, the user interface, and the data storage implementation.

\section{Data storage implementation}

The first task was to design a way to store the application data. The key requirements were user-friendly data update, and ability to save any place as favorite for particular user. Both Android and iOS have their own mechanisms for application data storage. For Android, it is a SQLite database and for iOS it is Core Data framework. Related problem is the synchronization of the data at the time of an update.

To resolve the second problem a decision was made to update the application data over a platform vendor through a regular update of the application. This approach has several benefits for developers as well as for the administrators. First of all, there is no need to have a separate server for data distribution. The update process is provided by the standard method which user is already accustomed for. The frequency of the updates also increases the app's rating in the application store and makes it easier for the user to find it.

After the agreement on the update mechanism, it was necessary to find a suitable approach for update of the internal database. Let us consider Android platform as an example. The update process is defined by the database version. The programmer has several alternative methods for updating the internal database when an application is upgraded. For example, it is possible to process data from a KML file stored in

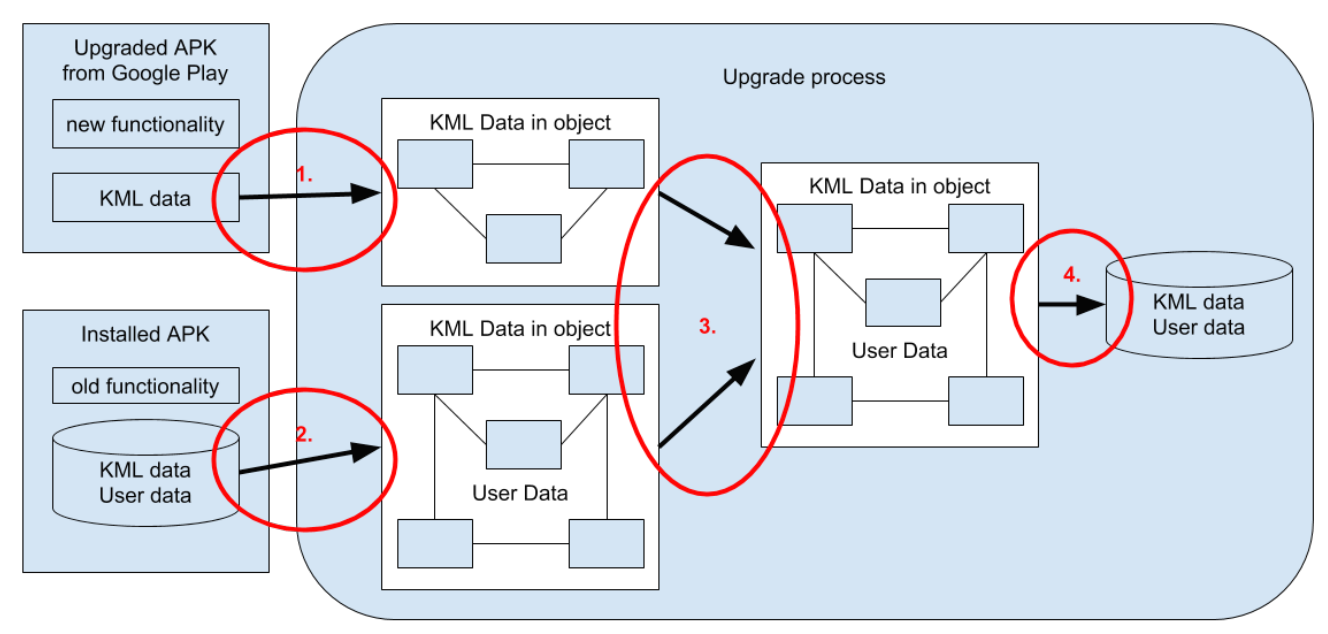

1: Schema of standard data transformation when updating the application. 
a resources into an internal database. However, this process includes data synchronization issues. A new version of the application has to import new data but persist not changed data too. Because we need to consider that a user can store its own data related to objects. This data should not be lost during an upgrade.

The standard recommended solution contains four operations (see Fig. 1):

- The geographical objects are parsed from raw data stored in a KML file.

- The raw data are transferred to objects which are suitable for saving to the local database.

- The two identical models are merged. This is the most difficult operation, which includes a pairing of objects.

- Finally, merged objects are saved into the database.

\section{User interface}

The mobile application was designed as a map-based location-based service. This means, that the key element of the application is a map. A user can select any object on the map and get the related information. Because the application was created as a native one, the user interface differs between the platforms. The user interface for both platforms can be seen in Fig. 2.

The filter is designed as a two-level search. A user firstly selects the primary category, e.g. playground. After the selection, the map points are immediately limited to places that belongs to the primary category. Subsequently, the user can choose from many secondary categories which will again result in the limitation of places (see Fig. 3).

\section{DISCUSSION}

As mentioned, the key challenge of our solution was to define a way for simple spatial data creation and updates without the need for a complex data server infrastructure. Usage of the KML files proved to be a very effective way for such updates by the city district officials. Moreover, the use of the KML file has one huge advantage. If the district decides to add a new type of location e.g. historical sites, it will be easy to implement such extension of the applications and only a new KML file needs to be provided.

However, absence of the server infrastructure also brought some disadvantages. The most important one is larger size of the final application. Because the images of places are stored locally on the device, the application becomes large and should be downloaded from the store only using Wi-Fi connection.

During the user testing phase, we received several tips from the testers. The first one was to include an initial help screen. The users had problems interacting with the map and the filter, so a help screen was implemented.
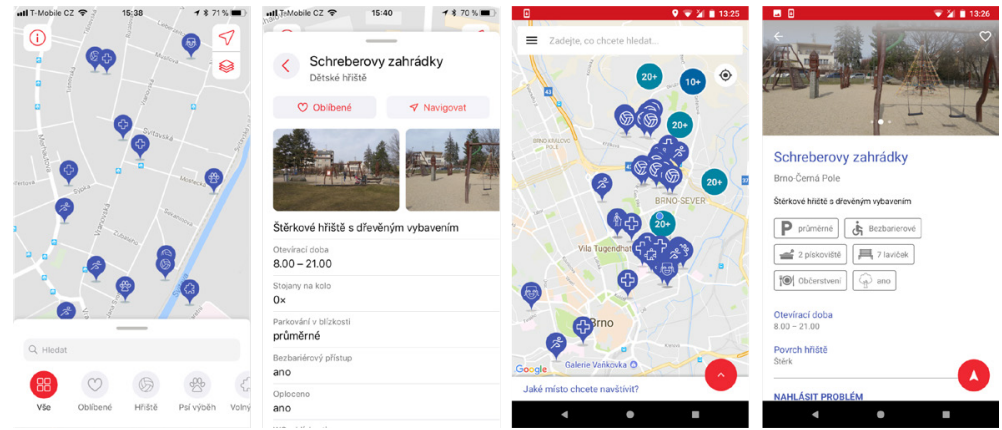

2: Screen shots from the mobile application. Left: iOS, right: Android
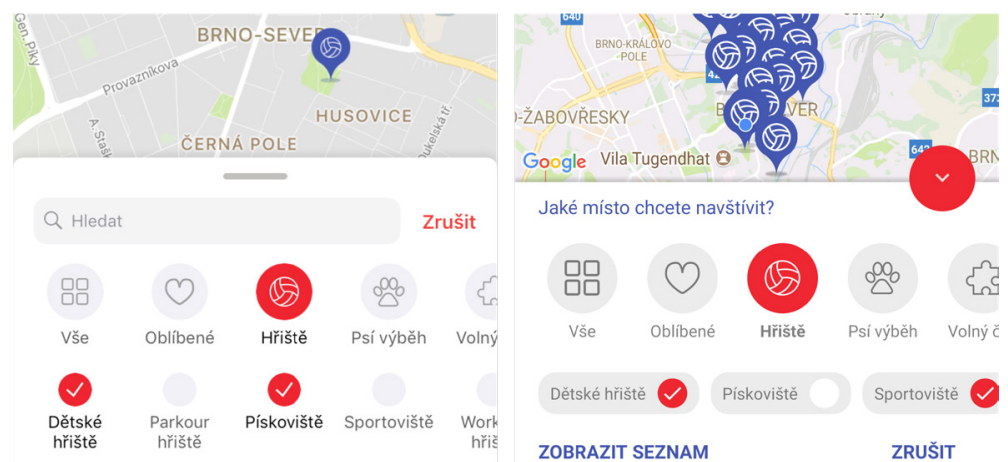

3: The place filter. The top row represents primary categories; the bottom row is used to select secondary categories. Left: iOS, Right: Android. 


\section{CONCLUSIONS}

This article describes the creation of location-based application for city district. The main goal of the application is to provide the citizens with a complete list of children playgrounds, dog runs and organizations providing services for families located in the city district. The application has been published to both Google Play and Apple App Store.

Important outcome of our work is the data collection. For the development of our application, the city district was forced to create a complete inventory of their playgrounds, dog runs and to create a complete list of all organizations providing services for families.

At this moment, the application is only in Czech; however, localizations into further languages are prepared for both platforms.

Acknowledgements

This work was supported by grant IGA FBE_TP_2017006 (SmartPEF: smart faculty).

\section{REFERENCES}

BILGIN, C. 2016. Mastering Cross-Platform Development with Xamarin. Packt Publishing.

COWLEY, R., JOSS, S., DAYOT, Y. 2018. The smart city and its publics: insights from across six UK cities. Urban Research and Practice, 11(1): 53-77.

DONGWOOK, K. and SUNGBUM, K. 2017. The Role of Mobile Technology in Tourism: Patents, Articles, News, and Mobile Tour App Reviews. Sustainability, 9(11): 2082.

GOOGLE. 2018. Google Places API. [Online]. Available at: https://developers.google.com/places/ [Accessed: 20 February 2018].

LEE,G.A.,DÜNSER,A.,KIM,S.andBILLINGHURST,M.2012.CityViewAR:A mobileoutdoorARapplication for city visualization. In: 2012 IEEE International Symposium on Mixed and Augmented Reality. 5-9 Nov. 2012, Atlanta, GA, USA. Available at: https://ir.canterbury.ac.nz/bitstream/handle/10092/8693/12642991_ ismar2012-lee-cityviewar\%20(2).pdf [Accessed: 21 February 2018].

LOWDERMILK, T. 2013. User-centered design [a developer's guide to building user-friendly applications]. Beijing: O'Reilly Media.

SCOTT, M. 2014. Old World, New Tech. The New York Times. [Online]. Available at: https://www.nytimes. com/2014/04/22/business/energy-environment/europe-remains-ahead-of-us-in-creating-smart-cities. html [Accessed: 18 February 2018].

Jaromír Landa: jaromir.landa@mendelu.cz Ivo Pisařovic:xpisarov@node.mendelu.cz Jan Kolomazník: jan.kolomaznik@mendelu.cz Ondřej Švehla: ondrej.svehla@mendelu.cz David Procházka:david.prochazka@mendelu.cz 